\title{
Lineage-specific early complete donor chimerism and risk of relapse after allogeneic hematopoietic stem cell transplantation for acute myeloid leukemia
}

\author{
Hannes Lindahl (iD ${ }^{1,2 \bowtie}$, Sofie Vonlanthen ${ }^{1}$, Davide Valentini ${ }^{3}$, Andreas T. Björklund ${ }^{3}$, Mikael Sundin (iD ${ }^{4,5}$, Stephan Mielke (iD ${ }^{6}$ and \\ Dan Hauzenberger ${ }^{1}$
}

(C) The Author(s) 2022

Recipient-donor chimerism is routinely analyzed after allogeneic hematopoietic stem cell transplantation (HSCT) to monitor engraftment and graft rejection. For malignancies, chimerism can also be used to screen for disease relapse post-HSCT but methodology and interpretation of results are not standardized and likely depend on underlying diagnosis. We have implemented highly sensitive and accurate methodologies for chimerism analysis for the purpose of improving relapse prediction. Here, we report an exploratory retrospective analysis of clinical routine chimerism results from all 154 HSCTs for acute myeloid leukemia (AML) performed at our center during the years 2015-2020 with the aim of suggesting a clinically useful threshold at which risk of relapse is high. Relapse was not reliably predicted based on single elevated chimerism values obtained before time of overt relapse. However, early complete donor chimerism, here defined as recipient DNA $<0.2 \%$ in $C D 33^{+}$cells in any blood or bone marrow sample taken during the first 60 days after HSCT, correlated inversely with relapse during the observation time (log-rank test $P=$ 0.033). We propose that achievement of complete chimerism determined early after HSCT using sensitive methods can be used for risk-stratification of AML patients.

Bone Marrow Transplantation (2022) 57:753-759; https://doi.org/10.1038/s41409-022-01615-8

\section{INTRODUCTION}

Allogeneic hematopoietic stem cell transplantation (HSCT) is a potentially curative treatment for hematological malignancies. Acute myeloid leukemia (AML) is the most common form of leukemia in adults and is a common indication for allogeneic HSCT [1]. Three-year overall survival after transplantation varies depending on AML risk profile but is on average less than $60 \%$ in Europe $[2,3]$. The prognosis after a post-HSCT AML relapse has improved somewhat over time but still remains poor [4]. Impending relapse may respond to treatment, primarily using donor lymphocyte infusion (DLI) or tapering of immunosuppression and in certain cases chemotherapy, second transplantation, or immune therapy $[5,6]$. Immune therapies currently under investigation are expected to further increase the treatment options $[7,8]$. Consequently, there is a large clinical need for improved early detection of relapse after allogeneic HSCT.

Mixed recipient-donor chimerism has repeatedly been associated with relapse of AML after allogeneic HSCT in both adult and pediatric patients, but how the individual values should be interpreted in clinical practice remains unclear [9]. Ideally, monitoring of relapse after allogeneic HSCT should be done as frequently as is practical to identify and treat potential relapses as early as possible. Relapse prediction after allogeneic HSCT is currently performed either by detection of minimal residual disease (MRD), using flow cytometry or real-time quantitative polymerase chain reaction (qPCR), or by estimating the proportion of recipient DNA i.e. chimerism [10]. AML is genetically heterogeneous and detection of tumor specific MRD markers is often not possible, and markers may be lost as disease evolves potentially leading to relapse in MRD marker-negative patients [11, 12]. Whereas MRD analysis is mainly performed on bone marrow samples, chimerism is readily applied to blood samples making frequent sampling more feasible. Inherent to the method, chimerism is only applicable in the context of allogeneic transplantations but can be used in essentially all leukemia subtypes. Apart from relapse, chimerism is also used to assess engraftment and graft rejection, which is a benefit but also complicates interpretation.

Chimerism analysis by short tandem repeats (STR)-PCR is established as the gold standard $[13,14]$. Although sensitivity is limited to $1-5 \%$ recipient DNA, increasing values has been associated with risk of relapse [15]. Chimerism-guided intervention has shown promising results of increased overall survival [9]. To achieve higher sensitivity, qPCR-based methods were developed

\footnotetext{
${ }^{1}$ Clinical Immunology and Transfusion Medicine, Karolinska University Hospital, Stockholm, Sweden. ${ }^{2}$ Department of Clinical Neuroscience, Karolinska Institutet, Stockholm, Sweden. ${ }^{3}$ Department of Cellular Therapy and Allogeneic Stem Cell Transplantation (CAST), Karolinska University Hospital, Stockholm, Sweden. ${ }^{4}$ Pediatric Hematology, Immunology and HCT, Astrid Lindgren Children's Hospital, Karolinska University Hospital, Stockholm, Sweden. ${ }^{5}$ Pediatrics, CLINTEC, Karolinska Institutet, Stockholm, Sweden. ${ }^{6}$ Department of Cell Therapy and Allogeneic Stem Cell Transplantation (CAST), Department of Laboratory Medicine (LabMED), Karolinska University Hospital and Institutet, Karolinska Comprehensive Cancer Center, Stockholm, Sweden. ${ }^{\circledR}$ email: Hannes.lindahl@ki.se
} 
Table 1. Patient and transplantation characteristics.

\begin{tabular}{|c|c|c|c|c|}
\hline & All & With relapse & No relapse & $P$ \\
\hline Transplants & 154 & $37(24)$ & $117(76)$ & \\
\hline Age at HSCT in y, median(range) & $51(4-73)$ & $50(5-73)$ & $49(4-73)$ & NS \\
\hline Male & $92(60)$ & $20(54)$ & $72(62)$ & NS \\
\hline \multicolumn{5}{|l|}{ Type of conditioning } \\
\hline Myeloablative & $108(71)$ & $26(70)$ & $82(70)$ & NS \\
\hline Reduced intensity & $45(29)$ & $10(27)$ & $35(30)$ & NS \\
\hline Identical sibling & $41(28)$ & $12(32)$ & $29(25)$ & NS \\
\hline Haploidentical related & $11(7)$ & - & $11(9)$ & NS \\
\hline Matched unrelated & $102(68)$ & $25(68)$ & $77(66)$ & NS \\
\hline \multicolumn{5}{|l|}{ Stem cell source } \\
\hline Peripheral blood & $135(88)$ & $33(89)$ & $102(87)$ & NS \\
\hline Days of follow-up, median (range) & $1138(99-2233)$ & $1413(281-2206)$ & $1029(99-2233)$ & 0.016 \\
\hline
\end{tabular}

Unless otherwise indicated, data are shown as $n(\%)$.

$P$ values for comparisons of transplants with relapse and without relapse are calculated using the $\chi 2$ test or unpaired students $T$-test as applicable. NS Not statistically significant, HSCT Hematopoietic stem cell transplantation, DLI Donor lymphocyte infusion.

[16] and studies indicate better performance in predicting relapse using serial testing in peripheral blood [17]. However, qPCR lack precision throughout the range of detection [18, 19]. We and others therefore used the two methods in parallel for some time. More recently, next generation sequencing (NGS) assays have been developed, which combine the sensitivity of qPCR assays and the precision of STR-PCR $[19,20]$. Even greater sensitivity can potentially be achieved by determining chimerism values for each of the major cell types separately by sorting cells prior to DNA extraction, as recipient DNA may not be detectable in all cell types leading to dilution of the signal. There are reports suggesting that even low absolute values of chimerism or subtle increments over time may predict relapse $[17,21]$. For this reason, we argue that a method, or combination of methods, that achieves both high sensitivity and accuracy is required to harness the full potential of chimerism analysis for relapse prediction.

Here, we report retrospective data on all allogeneic HSCTs for AML at Karolinska University Hospital in Sweden during the years 2015-2020 with the aim of suggesting a clinically useful threshold at which the risk of relapse motivates increased monitoring of the individual patient and potentially also preemptive interventions.

\section{METHODS}

\section{Patients and HSCTs}

This study was approved by the Swedish Ethical Review Authority and was conducted in accordance with the Declaration of Helsinki. Informed consent was obtained from all included patients. All consecutive HCSTs for AML performed at Karolinska University Hospital from 2005 until the end of 2020 were included. Clinical data incorporated in the European Society for Blood and Marrow Transplantation (EBMT) registry were used. Patient and transplant characteristics are presented in Table 1. Subclassification of included AML diagnoses are presented in table S1. The conditioning regimens varied and have been categorized as myeloablative conditioning and reduced intensity conditioning according to established criteria [22].

\section{Cell separation}

Cells were routinely separated into the major cell types using Dynabeads (ThermoFisher, Waltham, MA, USA) coupled to antibodies, generating separate results for bone marrow cells expressing CD3, CD19, CD33, or CD34 and in the case of blood, for cells expressing CD3, CD19, or CD33. For routine separation, $4 \mathrm{ml}$ of blood is processed but for small children as little as $2 \mathrm{ml}$ has been successfully used. For bone marrow, $3 \mathrm{ml}$ sample volume is requested but as little as $1 \mathrm{ml}$ has been processed successfully. Samples are generally processed within $24 \mathrm{~h}$ but can be stored up to $48 \mathrm{~h}$ at $4{ }^{\circ} \mathrm{C}$ before proceeding with the cell separation. Phosphate buffered saline (PBS) is added to a total volume of $5 \mathrm{ml}$. In the Arrow robot, $30 \mu \mathrm{l}$ of washed beads are added followed by a 15 -min incubation. Beads are then separated using a magnet and the negative fraction is used for subsequent separations. For blood samples, the cell separations proceed in the following order: $C D 19, C D 3$, and $C D 33$. For bone marrow samples, the original sample is split in two of which one is used for CD19 and CD3 and the other for CD34 and CD33 separation (in that order). The positive fractions are washed at least three times in PBS before proceeding with cell lysis and DNA extraction. The purity of cell separations is not routinely assessed but has been tested during the validation of the methods. The purity for blood samples was generally $>95 \%$ and for bone marrow samples $70-90 \%$.

\section{Chimerism}

The majority of the chimerism results were generated by STR-PCR quantification using capillary electrophoresis or qPCR. Both methods included prior screening of recipient and donor DNA to find informative markers, for which one (STR-PCR) or two (qPCR) were used for chimerism analysis of post-HSCT samples. The qPCR method was based on Taqman probes (ThermoFisher) of single nucleotide polymorphisms (SNP) or indels at 24 different loci. Routinely, STR-PCR was chosen for samples with higher amounts of mixed chimerism, taking advantage of the method's good accuracy, and qPCR was chosen for samples with low amounts of mixed chimerism, taking advantage of the method's good sensitivity [18]. In 2020, these two methods were replaced by a NGS-based method (Devyser AB, Stockholm, Sweden), which has both good accuracy throughout the analytical range and good sensitivity [19]. Chimerism is reported as percentage of recipient DNA throughout. Samples that did not generate a reliable chimerism result due to technical issues were removed in this study. Bone marrow and blood samples for recipient chimerism analysis were taken at the clinician's discretion and sampling frequencies are not uniform (Table 2). Consequently, patients were excluded when chimerism results were unavailable at time points and/or from sample types of interest for the analyses. 
Table 2. Chimerism data.

\begin{tabular}{lcccc} 
& Bone marrow & & Blood \\
& relapse & No relapse & relapse & No relapse \\
\hline Samples per patient & $3(2-7)$ & $6(4-8)$ & $3(2-4)$ & $2(1-4)$ \\
\hline Patients with chimerism data, $n(\%)$ & $33(89)$ & $102(90)$ & $34(92)$ & $103(91)$ \\
\hline Sampling interval before relapse, days & $50(30-67)$ & - & $52(31-113)$
\end{tabular}

Unless otherwise indicated data are shown as median (IQR).

$I Q R$ Interquartile range.

Table 3. Relapse predicted by max recipient chimerism $\left(\%_{\max }\right)$ in samples taken before or at time of relapse.

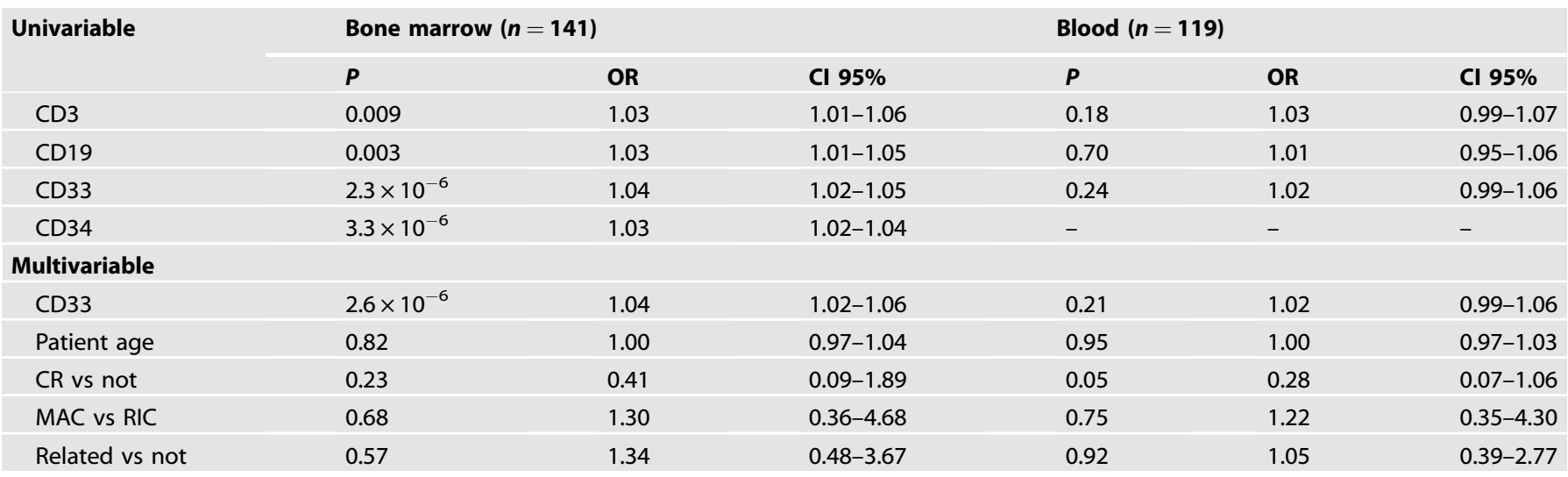

$P$ values are from logistic regression analysis.

$O R$ Odds ratio, $C l$ Confidence interval, $C R$ Complete remission before transplantation, MAC Myeloablative conditioning, RIC Reduced intensity conditioning,

Related vs not HLA-identical sibling or haploidentical relative vs matched unrelated donor, $n$ number of transplants.

\section{Statistical methods}

Descriptive statistics of patient characteristics and HSCT regimens are reported for the combined data set as well as separately for those that had a relapse and those that had no relapse during the study time. Four patients had their first and second HSCT within the study time making the total number of patients in the study fewer than the total number of transplantations. However, for all statistical models each transplantation is treated equally. Groups were compared using the $x^{2}$ test for categorical data and unpaired students $t$-test for numerical data. Simple and multiple logistic regression were used to assess the relationship between relapse and chimerism values as well as other independent variables that were considered potential confounders. Receiver Operating Characteristics (ROC) curve analysis was used to determine discriminative performance of the models and the optimal cutoff was determined using the Youden's J statistic (Youden's index). Relapse-free survival was calculated with the Kaplan-Meier method and the log-rank test was used for comparisons of curves. Correlations for non-normally distributed data were calculated using Spearman's rank correlation coefficient. $P$ values are two sided and 0.05 was considered statistically significant. All calculations and figures were performed using $R$ v 3.6.1 (the Comprehensive R Archive Network project) with RStudio v. 1.1.456 and the packages ggplot2, ROCit, and survminer.

\section{RESULTS}

Patients and samples

A total of $154 \mathrm{HSCT}$ s were performed for 150 patients with AML during 2015-2020 (Table 1). Included AML subtypes are listed in table S1. The study includes both pediatric $(n=10)$ and adult patients $(n=140)$ and the median age was 51 years (range, 4-73 years). The median follow-up time was 1,138 days (range 99-2,233). Thirty-seven transplants (24\%) resulted in a relapse within the study time after a median of 75 days post-HSCT. No statistically significant association between relapse and age, sex, type of conditioning, donor type, or stem cell source was observed
(Table 1). Relapse was however strongly associated with death. All available chimerism data for these patients were extracted, in total 5009 values from 1399 unique biological samples (Table 2). Whenever a bone marrow and blood sample were taken on the same day for chimerism analysis, these were considered as paired samples and the correlation between obtained values from these samples were plotted (Fig. S1).

\section{Mixed chimerism and prediction of relapse}

First, we assessed how well individual chimerism values can predict relapse if anticipation is not required. Chimerism results were filtered to remove samples that (1) were taken during the first 30 days after HSCT because mixed chimerism during this time most likely reflects engraftment (2) were taken after relapse or (3) were taken in the non-relapse group later than the average time to relapse in the relapse group, to adjust the observation time in the two groups. The variable $\%_{\max }$ was defined as the highest chimerism value for each transplantation during this time window (30 to on average 485 days after HSCT). 619 bone marrow samples from 141 transplants, of which 33 led to a relapse, were available. Thirteen transplants were thus excluded because no bone marrow sample for chimerism was taken during this time window. Univariable analysis showed a statistically significant association between relapse and $\%_{\max }$ recipient chimerism in bone marrow samples, which was most pronounced in $\mathrm{CD}_{3}{ }^{+}$cells (Table 3 ). Multivariable analysis showed that this association was independent of other plausible predictive factors of relapse. At relapse, $\mathrm{CD}_{3}{ }^{+}$and $\mathrm{CD}_{3} 3^{+}$cell chimerism were essentially correlated but a subset of patients had distinctly higher chimerism in $\mathrm{CD}_{3} 4^{+}$cells (Fig. 1). For $\%_{\max }$ in bone marrow $\mathrm{CD}^{+} 3^{+}$cells, receiver-operating characteristics (ROC)-analysis resulted in an area under the curve (AUC) of 0.83 (Fig. 2). Youden's index suggested an optimal cutoff at $6.3 \%$, which resulted in a sensitivity of $76 \%$ and a specificity of 
a

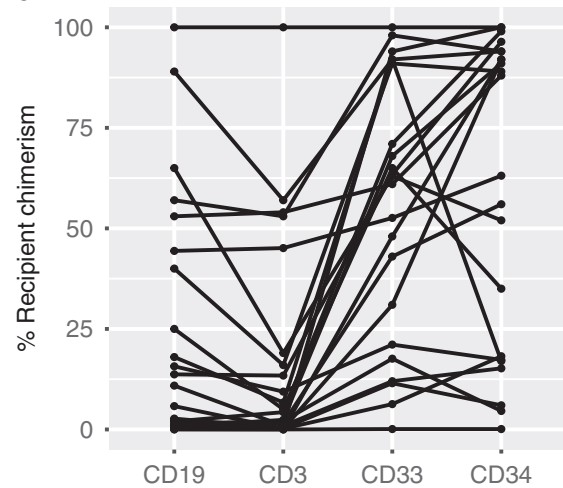

b

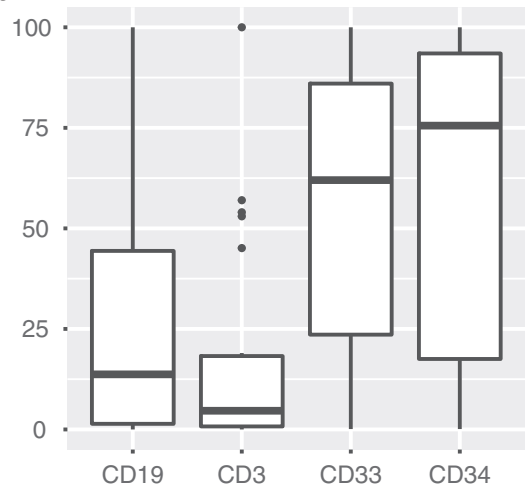

Fig. 1 Chimerism results from bone marrow samples taken at relapse. Percentage of recipient chimerism in four sorted cell fractions from bone marrow samples taken at the time of hematological relapse shown as (a) dots where individual patients are connected by lines and (b) boxplots representing the median and interquartile range.
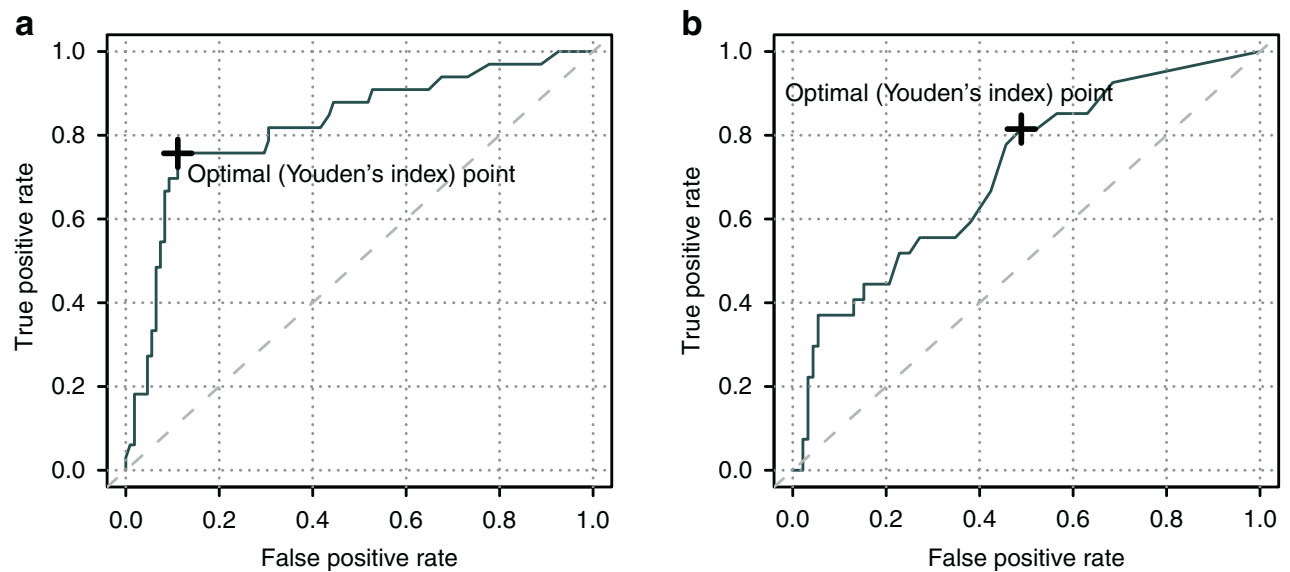

Fig. 2 Discrimination power of single chimerism values in $\mathrm{CD}_{33^{+}}$cells to predict impending or overt relapse. Receiver operating characteristics (ROC) curves depicting the true positive rate (sensitivity) and false positive rate (1-specificity) of relapse prediction in acute myeloid leukemia patients after hematopoietic stem cell transplantation. Chimerism from (a) bone marrow and (b) blood samples were analyzed at the clinician's discretion. The analysis includes chimerism values obtained from 30 days after transplantation to the day of clinical relapse or an equal length of time in the group that did not have a relapse. Youden's index suggest a cutoff that makes a trade-off between sensitivity and specificity.

89\%. The same analysis was then performed using the chimerism values from blood samples. After removing transplants without blood chimerism values ( $n=20), 134$ remained of which 26 led to a relapse. In contrast to bone marrow, univariable analysis did not result in a statistically significant association between $\%_{\max }$ in blood samples and relapse in any of the three sorted cell populations (Table 3). However, the number of blood samples were only 273 compared to 619 bone marrow samples, reflecting the practice at our center.

To assess whether individual chimerism values in this data set can predict relapse in advance, thus enabling preemptive measures to restrain the tumor cells, we excluded chimerism values at the time of overt relapse. In patients that did not have a relapse we excluded values that were taken later than the mean time from HSCT to last sample before overt relapse in the relapse group. Again, samples from the first 30 days after HSCT were removed. For the analysis of bone marrow samples, this left 503 chimerism values from 134 transplants of which 26 led to a relapse and for blood samples, 232 chimerism values from 118 transplants of which 26 led to a relapse. However, no statistically significant association between relapse and $\%_{\max }$ in any sample or cell type was observed (Table S2). The median number of samples per patient that remained after the filtration steps were four and two for bone marrow and blood, respectively.
Early complete donor chimerism and prediction of relapse Next, we investigated if low recipient chimerism values early after HSCT predicts a relapse-free disease course aiming to gain prognostic information related to the graft rather than to detect recurring disease. Analogous to previous analyses we defined the variable $\%_{\min }$ as the lowest chimerism value obtained during the first 60 days after HSCT. Bone marrow sampling during this time after HSCT is infrequent at our center. We therefore first analyzed only chimerism values from blood samples and then combined all values from both compartments for an additional analysis. For blood samples, univariable and multivariable analyses demonstrated a statistically significant association between future relapse and $\%_{\min }$ in $\mathrm{CD}_{3}{ }^{+}$cells (Table S3). Similar results were obtained if chimerism values from both blood and bone marrow were included, implying that results from both compartments are informative for the purpose of predicting future relapse. We continued with the combined chimerism values from $\mathrm{CD}_{3}{ }^{+}$blood and bone marrow cells and performed a ROC-curve analysis. The discriminative efficacy for $\%_{\min }$ to predict future relapse was modest, with an AUC of 0.63 . Youden's index suggested an optimal cutoff of $0.2 \%$, which resulted in a sensitivity of $50 \%$ and a specificity of $75 \%$. Next, we investigated if early complete chimerism using this low threshold of recipient DNA may be a useful means to stratify patients 
Table 4. Relapse predicted by complete donor chimerism ( $<0.2 \%$ recipient) in samples taken the first 60 days after HSCT.

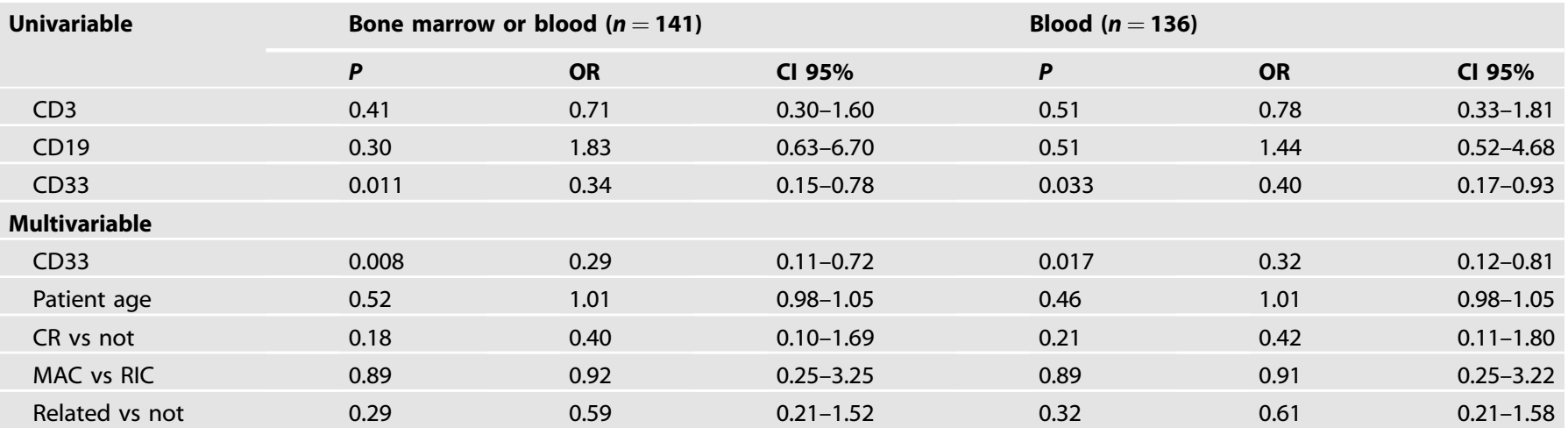

$P$ values are from logistic regression analysis.

$O R$ Odds ratio, $\mathrm{Cl}$ Confidence interval, $C R$ Complete remission before transplantation, MAC Myeloablative conditioning, RIC Reduced intensity conditioning, Related vs not HLA-identical sibling or haploidentical relative vs matched unrelated donor, $n$ Number of transplants.

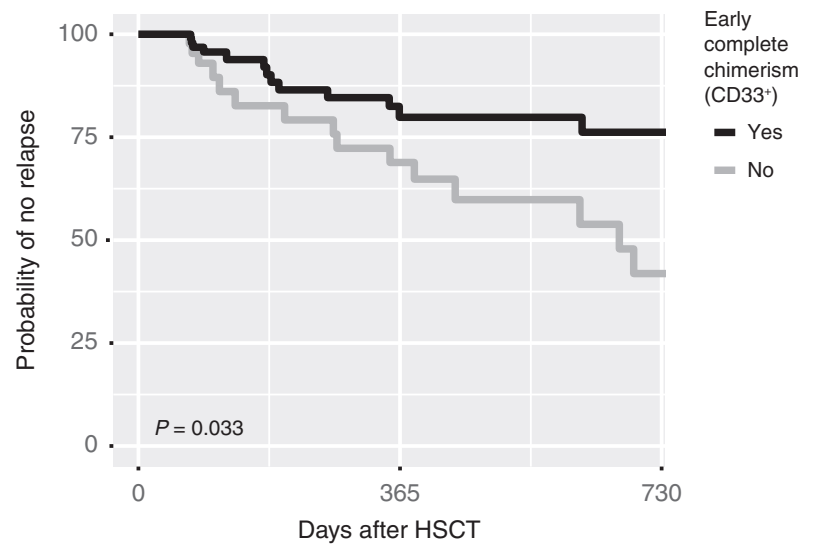

Fig. 3 Complete chimerism within the first 60 days after HSCT is an early indicator of relapse risk for patients with acute myeloid leukemia. The lowest chimerism value in $\mathrm{CD}_{3} 3^{+}$cells from blood or bone marrow sampled during the first 60 days after hematopoietic stem cell transplantation (HSCT) was used to stratify patients based on if early complete chimerism was achieved, here defined as having $<0,2 \%$ recipient DNA at any time during the first 60 days after HSCT. The two groups were compared using Kaplan-Meier estimates regarding relapse-free disease course.

as having high or low risk of relapse. We dichotomized all patients based on if early complete chimerism was reached, defined as at least one chimerism value below $0.2 \%$ in either blood or bone marrow $\mathrm{CD}_{3}{ }^{+}$cells in the first 60 days after HSCT. Patients that had a relapse within the first 60 days after HSCT were excluded from this analysis $(n=2) .141$ Patients of which 30 had a relapse were included. Early complete chimerism was observed in $50 \%$ of patients that later had a relapse and $75 \%$ of patients that did not have a relapse during the study time $\left(X^{2} P=0.017\right)$. Univariable and multivariable analysis was performed and showed a statistically significant association between early complete chimerism and future relapse (Table 4). Kaplan-Meier analysis showed a statistically significant lower probability of relapse in patients with early complete donor chimerism (log-rank test $P=0.033$ ) (Fig. 3). The probability of being relapse-free two years after HSCT for patients that achieved early complete chimerism was estimated at 0.76 (95\% Cl 0.65-0.90) compared to $0.42(0.25-0.70)$. The same Kaplan-Meier analysis performed on only blood $(n=135)$ or bone marrow $(n=44)$ samples showed similar trends but did not reach statistical significance (Fig. S2).

\section{DISCUSSION}

Relapse is the most common cause of death after allogeneic HSCT for AML [23] but treatments exist that may prevent a clinical relapse in its early stages [5-8]. Impending relapse can potentially be detected by tumor specific MRD markers or chimerism analysis. Chimerism has repeatedly been associated with relapse risk but cutoff values have not been established $[9,24]$. Our initial aim was to investigate in our retrospective data if increased recipient chimerism could be an early biomarker of an impending relapse and to suggest a cutoff value at which the risk is high. However, relapses in adults with AML often occur very rapidly and with the sampling frequency performed in clinical practice at our center, it was not possible to predict relapse with a sufficient degree of anticipation. Previous studies have suggested that more frequent chimerism analysis is required $[25,26]$. On the other hand, the data showed that failing to achieve early complete donor chimerism, using a very stringent threshold of $<0.2 \%$ recipient DNA in $\mathrm{CD}_{3}{ }^{+}$cells during the first 60 days after HSCT, was associated with increased risk of relapse and consequently has potential to be used for risk-stratification of AML patients post-HSCT.

Interestingly, chimerism in bone marrow and blood do not correlate reliably in our data (Fig. S1), which also has been observed by others [27-29] suggesting that the two compartments reflect different processes or have different kinetics. The observation that chimerism in bone marrow samples associated more strongly with relapse in our data than chimerism in peripheral blood samples may have a biological explanation and/or may be a consequence of less frequent sampling from peripheral blood. Monitoring chimerism in peripheral blood cells would be preferable because more frequent sampling is possible but based on our data the utility of peripheral blood chimerism analyses is difficult to assess and further elucidation of this would require a different study design.

At our center, we routinely perform chimerism analysis on fractionated cells to (1) achieve greater sensitivity and (2) add information about the graft composition. It has been shown that higher donor $\mathrm{T}$ cell chimerism in peripheral blood samples taken early after transplantation is associated with better prognosis [30]. In our data, we observed no statistically significant association between mixed chimerism in T cells and relapse. However, relapse was most consistently associated with mixed chimerism in the $\mathrm{CD}_{3} 3^{+}$fraction of bone marrow samples, reflecting the myeloid origin of the tumor cells. Chimerism in $\mathrm{CD}_{3} 4^{+}$was distinctly elevated in a large portion of patients at the time of overt relapse (Fig. 1), which may indicate a leukemic stem cell phenotype [31].

Donor chimerism gradually increases during the first days to weeks after allogeneic HSCT reflecting engraftment and graft alloreactivity. Various definitions of complete donor chimerism have 
been used, for example $<5-10 \%$ recipient. In most cases complete chimerism occurs within 30 days after $\operatorname{HSCT}[32,33]$. Previous retrospective studies of the utility of chimerism in AML patients post-HSCT have suggested that relapse can be predicted based on whether complete chimerism is achieved or not. One study defined complete chimerism as recipient DNA $<1 \%$ at any point in time after HSCT and only 4/75 patients failed to achieve this, of which three relapsed [34]. The largest retrospective study to date of the prognostic value of chimerism included relapse prediction as a secondary outcome [35] and included 193 AML patients. With a threshold of $10 \%$ recipient DNA, significant associations between chimerism in unsorted cells around day 30 as well as around day 100 with subsequent relapse were observed. In that study, chimerism was assessed by STR-PCR, which is less sensitive than QPCR or NGS and values from bone marrow and blood samples were analyzed together. Chimerism in $\mathrm{CD}^{+}$cells was analyzed in parallel but no added benefit from this was observed. Our data similarly suggest that assessment of complete chimerism early after allogeneic HSCT for AML has potential as a prognostic marker. Remaining recipientderived cells in either blood or bone marrow samples during this time window could serve as an indicator of the alloreactivity of the graft and thus efficiency of the graft vs leukemia effect. The association between low chimerism $\left(\%_{\mathrm{min}}\right)$ early post-HSCT and reduced risk of subsequent relapse was limited to the $\mathrm{CD}_{3}{ }^{+}$ fraction. This may reflect presence of remaining myeloid leukemia cells that have acquired a survival benefit compared to other recipient cells. Both $\mathrm{qPCR}$ and NGS are sensitive methods that reliably quantify chimerism $>0.01$ and $>0.1$, respectively [19]. With sensitive methods a low threshold can be applied that may increase the predictive properties of early complete donor chimerism.

In summary, achievement of complete donor chimerism assessed by sensitive methods early after HSCT for AML appears to be useful for relapse risk-stratification. The data suggest that patients that do not reach a stringently defined threshold of complete donor chimerism in blood or bone marrow samples during the first two months after HSCT should be monitored more closely. With the sampling frequency used at our center, mixed chimerism does not detect a developing relapse in its early stages but can confirm a manifested relapse. Questions that remain are whether mixed chimerism can be used to guide preemptive measure in the early stages of a relapse and whether analysis of blood samples has advantages over bone marrow samples because of the possibility of higher sampling frequencies. This should preferably be addressed in prospective studies.

\section{REFERENCES}

1. Passweg JR, Baldomero H, Bader P, Bonini C, Cesaro S, Dreger P, et al. Hematopoietic stem cell transplantation in Europe 2014: more than 40000 transplants annually. Bone Marrow Transpl. 2016;51:786-92

2. Lee CJ, Savani BN, Mohty M, Gorin NC, Labopin M, Ruggeri A, et al. Post-remission strategies for the prevention of relapse following allogeneic hematopoietic cell transplantation for high-risk acute myeloid leukemia: expert review from the Acute Leukemia Working Party of the European Society for Blood and Marrow Transplantation. Bone Marrow Transpl. 2019;54:519-530.

3. Ruggeri A, Labopin M, Ciceri F, Mohty M, Nagler A. Definition of GvHD-free, relapse-free survival for registry-based studies: an ALWP-EBMT analysis on patients with AML in remission. Bone Marrow Transpl. 2016;51:610-1.

4. Bazarbachi A, Schmid C, Labopin M, Beelen D, Wolfgang Blau I, Potter V, et al. Evaluation of trends and prognosis over time in patients with AML relapsing after allogeneic hematopoietic cell transplant reveals improved survival for young patients in recent years. Clin Cancer Res. 2020;26:6475-6482.

5. Platzbecker U, Wermke M, Radke J, Oelschlaegel U, Seltmann F, Kiani A, et al. Azacitidine for treatment of imminent relapse in MDS or AML patients after allogeneic HSCT: results of the RELAZA trial. Leukemia. 2012;26:381-9.

6. Schroeder T, Rautenberg C, Krüger W, Platzbecker U, Bug G, Steinmann J, et al. Treatment of relapsed AML and MDS after allogeneic stem cell transplantation with decitabine and DLI-a retrospective multicenter analysis on behalf of the German Cooperative Transplant Study Group. Ann Hematol. 2018;97:335-342.
7. Perl AE, Martinelli G, Cortes JE, Neubauer A, Berman E, Paolini S, et al. Gilteritinib or chemotherapy for relapsed or refractory FLT3-Mutated AML. N. Engl J Med. 2019;381:1728-1740.

8. Gong Y, Klein Wolterink RGJ, Wang J, Bos GMJ, Germeraad WTV. Chimeric antigen receptor natural killer (CAR-NK) cell design and engineering for cancer therapy. J Hematol Oncol. 2021;14:73.

9. Haugaard AK, Kofoed J, Masmas TN, Madsen HO, Marquart HV, Heilmann C, et al. Is microchimerism a sign of imminent disease recurrence after allogeneic hematopoietic stem cell transplantation? A systematic review of the literature. Blood Rev. 2020;44:100673.

10. Mo X-D, Lv M, Huang X-J. Preventing relapse after haematopoietic stem cell transplantation for acute leukaemia: the role of post-transplantation minimal residual disease (MRD) monitoring and MRD-directed intervention. $\mathrm{Br} J$ Haematol. 2017;179:184-197.

11. Xiao W, Petrova-Drus $K$, Roshal M. Optimal measurable residual disease testing for acute myeloid leukemia. Surg Pathol Clin. 2019;12:671-686.

12. Ofran $Y$, Rowe JM. Introducing minimal residual disease in acute myeloid leukemia. Curr Opin Hematol. 2015;22:139-45.

13. Antin $\mathrm{JH}$, Childs R, Filipovich AH, Giralt $\mathrm{S}$, Mackinnon $\mathrm{S}$, Spitzer T, et al. Establishment of complete and mixed donor chimerism after allogeneic lymphohematopoietic transplantation: recommendations from a workshop at the 2001 Tandem Meetings of the International Bone Marrow Transplant Registry and the American Society of Blood an. Biol Blood Marrow Transpl. 2001;7:473-85.

14. Clark JR, Scott SD, Jack AL, Lee H, Mason J, Carter Gl, et al. Monitoring of chimerism following allogeneic haematopoietic stem cell transplantation (HSCT): technical recommendations for the use of short tandem repeat (STR) based techniques, on behalf of the United Kingdom National External Quality Assessment Service. Br J Haematol. 2015;168:26-37.

15. Thiede $C$, Bornhäuser $M$, Oelschlägel U, Brendel $C$, Leo $R$, Daxberger $H$, et al. Sequential monitoring of chimerism and detection of minimal residual disease after allogeneic blood stem cell transplantation (BSCT) using multiplex PCR amplification of short tandem repeat-markers. Leukemia. 2001;15:293-302.

16. Sellmann L, Rabe K, Bünting I, Dammann E, Göhring G, Ganser A, et al. Diagnostic value of highly-sensitive chimerism analysis after allogeneic stem cell transplantation. Bone Marrow Transpl. 2018;53:1457-1465.

17. Jacque N, Nguyen S, Golmard J-L, Uzunov M, Garnier A, Leblond V, et al. Chimerism analysis in peripheral blood using indel quantitative real-time PCR is a useful tool to predict post-transplant relapse in acute leukemia. Bone Marrow Transpl. 2015;50:259-65.

18. Alizadeh M, Bernard M, Danic B, Dauriac C, Birebent B, Lapart C, et al. Quantitative assessment of hematopoietic chimerism after bone marrow transplantation by real-time quantitative polymerase chain reaction. Blood. 2002;99:4618-25.

19. Pettersson L, Vezzi F, Vonlanthen S, Alwegren K, Hedrum A, Hauzenberger D. Development and performance of a next generation sequencing (NGS) assay for monitoring of mixed chimerism. Clin Chim Acta. 2021;512:40-48.

20. Pedini $P$, Cherouat $N$, Basire A, Simon $S$, Budon $L$, Pourtein $M$, et al. Evaluation of next-generation sequencing and crystal digital PCR for chimerism monitoring of post-allogeneic hematopoietic stem cell transplantation. Transpl Cell Ther. 2021;27:89.e1-89.e10.

21. Haugaard AK, Madsen HO, Marquart HV, Rosthøj S, Masmas TN, Heilmann C, et al. Highly sensitive chimerism detection in blood is associated with increased risk of relapse after allogeneic hematopoietic cell transplantation in childhood leukemia. Pediatr Transpl. 2019;23:e13549.

22. Bacigalupo A, Ballen K, Rizzo D, Giralt S, Lazarus $H$, Ho V, et al. Defining the intensity of conditioning regimens: working definitions. Biol Blood Marrow Transpl. 2009;15:1628-33.

23. Horowitz $M$, Schreiber $\mathrm{H}$, Elder $\mathrm{A}$, Heidenreich $\mathrm{O}$, Vormoor J, Toffalori $\mathrm{C}$, et al. Epidemiology and biology of relapse after stem cell transplantation. Bone Marrow Transpl. 2018;53:1379-1389.

24. Huisman C, de Weger RA, de Vries L, Tilanus MGJ, Verdonck LF. Chimerism analysis within 6 months of allogeneic stem cell transplantation predicts relapse in acute myeloid leukemia. Bone Marrow Transpl. 2007;39:285-91.

25. Bader P, Beck J, Frey A, Schlegel PG, Hebarth H, Handgretinger R, et al. Serial and quantitative analysis of mixed hematopoietic chimerism by PCR in patients with acute leukemias allows the prediction of relapse after allogeneic BMT. Bone Marrow Transpl. 1998;21:487-95.

26. Thiede C, Bornhäuser M, Ehninger G. Strategies and clinical implications of chimerism diagnostics after allogeneic hematopoietic stem cell transplantation. Acta Haematol. 2004;112:16-23.

27. Stumph J, Vnencak-Jones CL, Koyama T, Frangoul H. Comparison of peripheral blood and bone marrow samples for detection of post transplant mixed chimerism. Bone Marrow Transpl. 2008;41:589-90.

28. Qin X-Y, Li G-X, Qin Y-Z, Wang Y, Wang F-R, Liu D-H, et al. Quantitative chimerism kinetics in relapsed leukemia patients after allogeneic hematopoietic stem cell transplantation. Chin Med J (Engl). 2012;125:1952-9. 
29. Waterhouse M, Pfeifer D, Duque-Afonso J, Follo M, Duyster J, Depner M, et al. Droplet digital PCR for the simultaneous analysis of minimal residual disease and hematopoietic chimerism after allogeneic cell transplantation. Clin Chem Lab Med. 2019;57:641-647.

30. Saito B, Fukuda T, Yokoyama H, Kurosawa S, Takahashi T, Fuji S, et al. Impact of T cell chimerism on clinical outcome in 117 patients who underwent allogeneic stem cell transplantation with a busulfan-containing reduced-intensity conditioning regimen. Biol Blood Marrow Transpl. 2008;14:1148-1155.

31. Jentzsch $M$, Bill $M$, Nicolet $D$, Leiblein S, Schubert K, Pless $M$, et al. Prognostic impact of the CD34+/CD38- cell burden in patients with acute myeloid leukemia receiving allogeneic stem cell transplantation. Am J Hematol. 2017;92:388-396.

32. Philip J, Bajaj AK, Sharma S, Kushwaha N, Kumar S, Kumar Biswas A. Allogeneic peripheral blood stem cell transplant: correlation of donor factors with yield engraftment, chimerism, and outcome: retrospective review of a single institute during a 3-year period. Lab Med. 2020;51:362-369.

33. Miura Y, Tanaka J, Toubai T, Tsutsumi Y, Kato N, Hirate D, et al. Analysis of donortype chimerism in lineage-specific cell populations after allogeneic myeloablative and non-myeloablative stem cell transplantation. Bone Marrow Transpl. 2006;37:837-43.

34. Wiedemann B, Klyuchnikov E, Kröger N, Zabelina T, Stahl T, Zeschke S, et al. Chimerism studies with quantitative real-time PCR in stem cell recipients with acute myeloid leukemia. Exp Hematol. 2010;38:1261-71.

35. Koreth J, Kim HT, Nikiforow S, Milford EL, Armand P, Cutler C, et al. Donor chimerism early after reduced-intensity conditioning hematopoietic stem cell transplantation predicts relapse and survival. Biol Blood Marrow Transpl. 2014:20:1516-21.

\section{AUTHOR CONTRIBUTIONS}

Conceptualization and design: $\mathrm{HL}$ and $\mathrm{DH}$; Collection and assembly of data: DV, SV ATB and MS; Data analysis and manuscript writing: $\mathrm{HL}$; Data interpretation and manuscript editing: $\mathrm{HL}, \mathrm{SV}, \mathrm{DV}, \mathrm{ATB}, \mathrm{MS}, \mathrm{SM}$ and $\mathrm{DH}$.

\section{FUNDING}

This work was supported by funding from the Department of Clinical Immunology and Transfusion Medicine, Karolinska University Hospital, Sweden. Open access funding provided by Karolinska Institute.

\section{COMPETING INTERESTS}

Dan Hauzenberger is founder and co-owner of Devyser AB. He is also part-time employed by the company. Stephan Mielke has received speakers fee from Novartis and DNA Prime SA, has received travel support from and served in expert panel for Gilead/KITE, has received travel support and speakers fee from Celgene/BMS, has received research funding, travel support, and speakers fee from Kiadis Pharma, has served in expert panel for Bellicum, has received travel support and speakers fee from and has served in data safety monitoring board for Miltenyi, and has served in data safety monitoring board for Immunicum.

\section{ADDITIONAL INFORMATION}

Supplementary information The online version contains supplementary material available at https://doi.org/10.1038/s41409-022-01615-8.

Correspondence and requests for materials should be addressed to Hannes Lindahl.

Reprints and permission information is available at http://www.nature.com/ reprints

Publisher's note Springer Nature remains neutral with regard to jurisdictional claims in published maps and institutional affiliations.

Open Access This article is licensed under a Creative Commons Attribution 4.0 International License, which permits use, sharing, adaptation, distribution and reproduction in any medium or format, as long as you give appropriate credit to the original author(s) and the source, provide a link to the Creative Commons license, and indicate if changes were made. The images or other third party material in this article are included in the article's Creative Commons license, unless indicated otherwise in a credit line to the material. If material is not included in the article's Creative Commons license and your intended use is not permitted by statutory regulation or exceeds the permitted use, you will need to obtain permission directly from the copyright holder. To view a copy of this license, visit http://creativecommons. org/licenses/by/4.0/.

(c) The Author(s) 2022 